\title{
GENERALIZED HADWIGER NUMBERS FOR SYMMETRIC OVALS
}

\author{
VALENTIN BOJU AND LOUIS FUNAR
}

(Communicated by Dale Alspach)

\begin{abstract}
Some estimations for the "juxtaposition function" $h_{F}$ and an asymptotic formula for the function $h_{F} / h_{G}$, where $F, G$ are central symmetric convex bodies, are given. Hadwiger and Grünbaum gave for $h_{F}(1)$ the bounds $n^{2}+n \leq h_{F}(1) \leq 3^{n}-1$. Grünbaum conjectured (and proved for $n=2$ in Pacific J. Math. 11 (1961), 215-219) that for every even $r$ between these bounds there exists in $E^{n}$ an oval $F$ such that $h_{F}(1)=r$. Lower bounds for $h_{F}$ could be derived in the same way as in Theorems 1 and 2 from a good estimate of packing numbers on a Minkowski sphere, that is, from solutions to a Tammes-type problem in a Banch space.
\end{abstract}

For a topological disk $F \subseteq \mathbb{E}^{n}$ we shall denote by $h_{F}:(0,1] \rightarrow \mathbb{N}$ the "juxtaposition function" introduced by the first author [2,3] as follows. Let $A_{F, \lambda}$ denote the family of all sets, homothetic to $F$ in the ratio $\lambda$, which have only boundary points in common with $F$. Then $h_{F}(\lambda)$ is the greatest integer $k$ such that $A_{F, \lambda}$ contains $k$ sets with pairwise disjoint interiors. In particular, $h_{F}(1)$ is just the Hadwiger number of $F$.

In case of convex $F$, Hadwiger [11] and Grünbaum [8] gave for $h_{F}(1)$ the bounds $n^{2}+n \leq h_{F}(1) \leq 3^{n}-1$. Grünbaum [8] conjectured (and proved for $n=2$; see also Boltjanski and Gohberg [4]) that for every even $r$ between these bounds there exists in $\mathbb{E}^{n}$ an oval $F$ such that $h_{F}(1)=r$.

Unless explicitly stated otherwise, throughout this paper $F, G$ will denote symmetric plane ovals. Any such $F$ determines a norm \|\|$_{F}$ by $\|x-y\|_{F}=$ $\|x-y\| /\|o-z\|$, where \|\| is the Euclidean norm, $o$ is the center of $F$, and $z$ is a point on the boundary $\partial F$ of $F$ such that $o z$ and $x y$ are parallel. With this norm $\mathbb{E}^{2}$ becomes a Banach space, with unit disk isometric to $F$. Set $p(F)$ for the perimeter of $\partial F$ in its inner norm.

Theorem 1. For a symmetric oval $F$ in the plane

$$
p(F)=2 \lim _{\lambda \rightarrow 0} \lambda h_{F}(\lambda) .
$$

Proof. Let $x, y$ be points of $\partial F$, and let points $x^{\prime}, y^{\prime}$ be given by $o x^{\prime}=$ $(1+\lambda) o x$ and $o y^{\prime}=(1+\lambda) o y$. Denote by $F_{x}, F_{y}$ those sets in $A_{F, \lambda}$ which have centers at $x^{\prime}$ and $y^{\prime}$, respectively. If $F_{x} \cap F_{y} \neq \varnothing$, it follows from the

Received by the editors March 17, 1992.

1991 Mathematics Subject Classification. Primary 52A15, 52A35.

(C) 1993 American Mathematical Society $0002-9939 / 93 \$ 1.00+\$ .25$ per page 
symmetry and convexity of $F$ that $x^{\prime} y^{\prime} \subset F_{x} \cap F_{y}$. We put $x^{\prime} y^{\prime} \cap \partial F_{x}=\{a, b\}$, $x^{\prime} y^{\prime} \cap \partial F_{y}=\{c, d\}$, and $z \in \partial F$ such that $o z$ is parallel to $x^{\prime} y^{\prime}$. Then

$$
\left\|x^{\prime}-y^{\prime}\right\| \leq\left\|x^{\prime}-b\right\|+\left\|c-y^{\prime}\right\|=2\left\|x^{\prime}-b\right\|=2 \lambda\|o-z\|,
$$

hence

$$
\|x-y\|_{F}=\left\|x^{\prime}-y^{\prime}\right\| /(1+\lambda)\|o-z\| \leq 2 \lambda /(1+\lambda) .
$$

Reversing the reasoning we obtain

$$
\text { int } F_{x} \cap \text { int } F_{y}=\varnothing \quad \text { if and only if }\|x-y\|_{F} \leq 2 \lambda /(1+\lambda) .
$$

Now consider a maximal collection $\left\{F_{i}: i=1, \ldots, k\right\} \subset A_{F, \lambda}$ of sets with disjoint interiors and the points $x_{i} \in \partial F, i=1, \ldots, k$, for which $F_{i}=F_{x_{i}}$. From (2) it follows that $\left\|x_{i}-x_{i+1}\right\|_{F} \leq 2 \lambda /(1+\lambda)$ and thus

$$
\sum_{1 \leq i \leq k}\left\|x_{i}-x_{i+1}\right\|_{F} \leq 2 k \lambda(1+\lambda)
$$

however,

$$
p(k, F)=\sup \left\{\sum_{1 \leq i \leq k}\left\|x_{i}-x_{i+1}\right\|_{F}, x_{i} \in \partial F\right\} \leq p(F) .
$$

These inequalities yield

$$
h_{F}(\lambda)=k \leq(1+\lambda) p(k, F) / 2 \lambda<(1+\lambda) p(F) / 2 \lambda .
$$

Conversely, let $P_{\lambda}$ be an inscribed polygon with $2 k$ vertices $u_{1}, \ldots, u_{2 k}$ such that $P_{\lambda}$ is symmetric about $o$ and

$$
\begin{gathered}
\left\|u_{1}-u_{2}\right\|_{F}=\left\|u_{2}-u_{3}\right\|_{F}=\cdots=\left\|u_{k-2}-u_{k-1}\right\|_{F}=2 \lambda /(1+\lambda), \\
2 \lambda /(1+\lambda) \leq\left\|u_{k-1}-u_{k}\right\|_{F}<4 \lambda /(1+\lambda) .
\end{gathered}
$$

Then the sets $F_{u_{i}}$ have disjoint interiors and

$$
4(k+1) \lambda /(1+\lambda)>\sum_{1 \leq i \leq 2 k}\left\|u_{i}-u_{i+1}\right\|_{F} \geq 4 k \lambda /(1+\lambda) .
$$

Since $h_{F}(\lambda) \geq 2 k$, it follows that

$$
2+h_{F}(\lambda) \geq(1+\lambda)\left(\sum_{1 \leq i \leq 2 k}\left\|u_{i}-u_{i+1}\right\|_{F}\right) / 2 \lambda .
$$

If $p(\lambda)_{F}$ denotes the perimeter of $P_{\lambda}$ in the \|\|$_{F}$ norm, then $($ see $[1,11])$

$$
\lim _{\lambda \rightarrow 0} p(\lambda)_{F}=p(F) .
$$

For symmetric ovals $F, G$ relations (3)-(5) imply

$$
\begin{aligned}
\lim _{\lambda \rightarrow 0} h_{F}(\lambda) / h_{G}(\lambda) & \geq \lim _{\lambda \rightarrow 0}\left(\left(-2+(1+\lambda) p(\lambda)_{F}\right) / 2 \lambda\right) /((1+\lambda) p(G)) / 2 \lambda \\
& =p(F) / p(G),
\end{aligned}
$$

and similarly the reverse inequality. Therefore, taking for $G$ a square we obtain the claim which was to be proved.

Denote by $[t]$ the integer part of $t \in \mathbb{R}$. 
Theorem 2. For every symmetric oval $F$ in the plane

$$
3+[3 / \lambda] \leq h_{F}(\lambda) \leq 4(1+\lambda) / \lambda,
$$

with equality on the left if and only if $1 / \lambda \in \mathbb{N}$, and $F$ is an affine-regular hexagon and equality on the right if and only if $1 / \lambda \in \mathbb{N}$ and $F$ is a parallelogram.

Proof. A result of Golab [6] and Reshetnyak [14], generalized by Schäffer [15], asserts that $6 \leq p(F) \leq 8$. Hence we have

$$
h_{F}(\lambda) \leq 4(1+\lambda) / \lambda,
$$

and, using the existence of an affine-regular hexagon inscribed in $F$ [13], we obtain $h_{F}(2 /(1+k)) \geq 6 k$. Since $h_{F}(\lambda)$ is a decreasing function of $\lambda$, we are done.

If the dimension of $F$ is greater than two, the situation is essentially different. We shall prove (see also [7])

Theorem 3. Any symmetric convex body $F \subset \mathbb{E}^{n}$ satifies the inequality

$$
h_{F}(\lambda) \leq\left((1+\lambda)^{n}-1\right) / \lambda^{n},
$$

with equality if and only if $1 / \lambda \in \mathbb{N}$ and $F$ is a parallelohedral body.

Proof. Let $B_{\lambda}=\bigcup_{H \in A_{F, \lambda}} H$. We shall prove that

$$
B_{\lambda} \subset(1+2 \lambda) F \text {. }
$$

Indeed, let $x$ be a point on the boundary of $F_{v},|o x| \cap \partial F=\{a\},|o v| \cap$ $\partial F_{v}=\{q\}$, and let $v x^{\prime \prime}$ be parallel to $q x$ with $x^{\prime \prime} \in \partial F$. Then $\measuredangle q v^{\prime} x=$ $\measuredangle v^{\prime} x o+\measuredangle v^{\prime} o x \geq \measuredangle v^{\prime} o x$, which yields $\measuredangle v o x \leq \measuredangle v o x^{\prime \prime}=\measuredangle q v^{\prime} x$. Since $F$ is convex, we can take a point $b$ in the nonempty intersection $|o a| \cap\left|v x^{\prime \prime}\right|$. Then $\left|v x^{\prime \prime}\right| \subset F, b \in F, b \in|o x|$. Since

$$
\|o+a\| /\|o-x\| \geq\|o-b\| /\|o-x\| \geq\|o-v\| /\|o-q\|=1 /(1+2 \lambda),
$$

the point $x$ belongs to $(1+2 \lambda) F$, and (9) is proved.

If $\left\{F_{i}, i=1, \ldots, k\right\} \subset A_{F, \lambda}$ have disjoint interiors, then

$$
\bigcup_{1 \leq i \leq k} F_{i} \subset B_{\lambda} \subset(1+2 \lambda) F
$$

therefore,

$$
\operatorname{vol}(F)+\operatorname{vol}\left(F_{1}\right)+\cdots+\operatorname{vol}\left(F_{k}\right) \leq(1+2 \lambda)^{n} \operatorname{vol}(F)
$$

where $\operatorname{vol}(F)$ denotes the volume of $F$. This gives the desired estimation on $h_{F}(\lambda)$. The equality case is treated in [7].

Lower bounds for $h_{F}(\lambda)$ could be derived in the same way as in Theorems 1 and 2 from a good estimate of packing numbers on a Minkowski sphere, that is, from solutions to a Tammes-type problem in a Banach space.

Grünbaum asked what happens to relation (1) in case $F$ is not centrally symmetric. We recall that for an arbitrary oval $F$ and $z \in \operatorname{int} F$ a norm (nonsymmetric, in general) is defined by the Minkowski functional

$$
\|x\|_{F, z}=\inf \{\lambda>0: x-z \in \lambda(F-z)\} .
$$


Using the (possibly nonsymmetric) distance derived from this norm it is possible to define arc-length for oriented arcs. For an oriented closed curve $C$ let the length of $C$ in the metric derived from \|\|$_{F, z}$ be denoted by $p_{F, z}(C)$. The intrinsic perimeter (self-circumference $[6,10])$ of $F$ is $P(F)=\inf \left\{p_{F, z}(\partial F)\right.$ : $z \in$ int $F\}$. Then it follows that

$$
g(F)=\lim _{\lambda \rightarrow 0} \lambda h_{F}(\lambda) / P(F)
$$

is a measure of symmetry (see [8]). By the same method as used above, it is possible to show that $g(F) \leq \frac{1}{2}$, with equality if $F$ is centrally symmetric. If $F$ is a triangle then $g(F)=\frac{1}{3}$, and we conjecture that $g(F) \geq \frac{1}{3}$ for any oval $F$.

\section{ACKNOWLEDGMENT}

We wish to thank Professor Branko Grünbaum for valuable suggestions, comments about this paper, and references.

\section{REFERENCES}

1. W. Blaschke, Kreis und Kugel, Veit, Berlin, 1916.

2. V. Boju, Fonctions de juxtaposition, Invariants, Proc. XIII Conf. Geom. Topology, Univ. Cluj-Napoca, 1982, pp. 36-37.

3. __ Courbures riemanniennes généralisées, Int. Conf. Geom. and Appl., Bulg. Acad. Sci., Sofia, 1986, pp. 47-48.

4. V. Boltjanski and L. Gohberg, Results and problems in combinatorial geometry, Mir, Moscow, 1965; English transl., Cambridge Univ. Press, 1985.

5. L. Danzer, B. Grünbaum, and V. Klee, Helly's theorem and its relatives, Proc. Sympos. Pure Math., vol. 7, Amer. Math. Soc., Providence, RI, 1963, pp. 101-180.

6. S. Golab, Quelques problemes métriques de la géometrie de Minkowski, Trav. Acad. Mines Cracov. 6 (1932). (Polish and French)

7. H. Groemer, Abschatzungen fur die Anzahl der konvexen Korper, die einen konvexen Korper beruhren, Monatsh. Math. 65 (1961), 74-81.

8. B. Grünbaum, On a conjecture of H. Hadwiger, Pacific J. Math. 11 (1961), 215-219.

9. __, Measures of symmetry for convex sets, Proc. Sympos. Pure Math., vol. 7, Amer. Math. Soc., Providence, RI, 1963, pp. 233-270.

10. __ Self-circumference of convex sets, Colloq. Math. 13 (1964), 55-57.

11. H. Hadwiger, Uber Treffanzahlen bei translationsgleichen Eikorpern, Archiv. Math 8 (1957), 212-213.

12. D. Laugwitz, Konvexe Mittelpunktsbereiche und normierte Raume, Math. Z. 61 (1954), 235-244.

13. K. Leichtweiss, Konvexe Mengen, VEB Deutsch. Verlag Wissenschaften, Berlin, 1980.

14. Y. G. Reshetnyak, An extremal problem from the theory of convex curves, Uspehi Math. Nauk 8 (1953), 125-126.

15. J. Schäffer, Inner girth of spheres, Math. Ann. 184 (1970), 169-171.

Department of Mathematics, University of Craiova, A. I. Cuza no. 13, Craiova 1100 , ROMANIA

InSTitute of MAThematics, P.O. BOX 1-700, 70700 Bucharest, RoMANiA

Current address, L. Funar: Université de Paris-Sud, Mathématiques, Bat. 425, 91405, Orsay Cedex, France

E-mail address, L. Funar: funar@matups.matups.fr 